\title{
SUGGESTIONS FOR BEHAVIOR-INTERVENTION DESIGN PRACTITIONERS: FROM BEHAVIOR CHANGE MOTIVATIONS FOR CHINESE AGED 18 TO 25
}

\author{
Yin, Yuan; \\ Yu, Yurong \\ Imperial College London
}

\begin{abstract}
Using applications to change behaviors is a popular trend in recent years as mobiles are the easiest recording medium for users. However, few users can keep the behavior change for a long time. The aim of this study is to investigate motivations of keeping an application-tracked behavior change to provide effective and promote effective and targeted suggestions for application-tracked behavior intervention design practitioners and researchers. A 28-day self-report experiment and following "focus group" discussion have been conducted to detect the possible motivations. The results indicated 8 motivations which can affect maintaining behavior change: cooperation, competition, award, reminder and alarm, trust and willingness, relation with disease information and unplanned events. In addition, the results explore some motivations from negative data in applications or the cheating for good performance data behavior. At the same time, the study suggested the functions needed in future behavior change applications.
\end{abstract}

Keywords: Digital behavior change, Motivations, Human behaviour in design, User centred design, Early design phases

Contact:

YIN, Yuan

Imperial College London

Dyson School of Design Engineering

United Kingdom

y.yin19@ic.ac.uk 


\section{INTRODUCTION}

Individual digital-evidence data can be collected when interacting with mobile applications (McGloin and Eslami, 2015). Although it may include ethical problems (Gubrium et al., 2014), smartphone applications are a feasible choice to track and record human behavior (Patrick et al., 2016). Since human behavior can be recorded, this triggers the thinking that if digital applications can intervene behaviors. This triggers the generation of digital behavior change intervention (DBCI), which is a process to trigger behavior change using an application on mobiles (Perski et al., 2019).

DBCIs are mainly based on remote technologies such as smartphones (Lathia et al., 2013), applications (Stockwell et al., 2019), websites (Roberts et al., 2017). As one of the reliable, stable, and feasible interventions, application-tracked behavior change is paid a high attention (Lathia et al., 2013). Invention designers try to improve the acceptability, usability, and satisfaction of the application-tracked behavior change intervention (Yardley et al., 2015). Some methods have also been applied to make the application-tracked behavior change work effectively, such as interaction, personality, motivation, positive experiment, credibility, and social connection (McGloin and Eslami, 2015; Morrison, 2015). "Motivation" is one of the effects that can persist in behavior change.

Therefore, the purpose of this paper is to detect the factors affecting motivations of maintaining DBCI. We tend to generate effective and targeted suggestions for application-tracked behavior intervention design practitioners and researchers from these factors.

The contribution of this study is that:

(1) We summarized eight factors that can affect motivations of maintaining DBCI for 18 to 25 aged Chinese participants.

(2) Based on the eight motivation factors summarized above, the paper promoted six targeted suggestions for application-tracked behavior interventions design practitioners. These implications can help designers build effective intervention for digital behavior change.

\section{LITERATURE REVIEW}

Some factors that can motivate DBCI have been promoted from existing research such as selfdetermination, social interaction, positive experience, award, alarm, and trust. Self-determination is a spontaneous motivation (Ryan and Deci, 2000). Currently, self-determination is applied to the DBCI design strategy. It can be enhanced through interventions such as exercising suggestions from doctors, environment, and competence. In other words, internal motivations are always stimulated by external stimulation. However, individual actions are highly influenced by the environment, especially the social environment (McGloin and Eslami, 2015; Rooksby et al., 2014). Social connection, including cooperation and competition, can effectively affect the intervention (Lathia et al., 2013; Morrison et al., 2012). For example, social connection can be maintained by communication applications, and users can obtain motivations from social applications (Elaheebocus et al., 2018). Cooperation has been identified as a method to motivate behaviors (Lathia et al., 2013; Mohr et al., 2011; Schueller et al., 2013). Many researchers have proved this, eg. Conroy, et al.(2014)detected how applications support health and fitness; Marshall, et al. (2003) and Park et al.(2008) used self-report to detect the process of physical activities. However, if sharing data would hinder the behavior change is not identified (Yardley et al., 2016). In addition, although much research on cooperation, there is less research on how competition, the other social connection, affects the motivation of digital behavior change in application.

A positive or expected positive experience can be considered a motivation(Morrison et al., 2012; Yardley et al., 2016). The positive user experience can be considered an award, such as being paid for achieving the goal (Conroy et al., 2014). Expected user experience is the predictable awards from dedication (Yang et al., 2015). However, which kinds of results or outputs are awards remains uncertain.

Some researchers agree that as an invaded life function, alarm will bring discomfort to users (Morrison et al., 2012; Yardley et al., 2016). Researchers also pointed out that even for those who think the alarm is less invaded, they still considered the alarm as a negative motivation effect (Lorig et al., 2008; Spittaels et al., 2007). However, different from the research, the fact is that nearly all behaviorchanging applications will involve the alarm function, which indicated that alarm might be a positive method to motivate behavior change. 
Trust is important (Michie et al., 2017), especially when the trust can be obtained from experts (Patten et al., 2007). For example, compared with other familiar members, it will be more persuasive if the statement about smoking would affect health is given by doctors (Patten et al., 2007; Yardley et al., 2016). However, some researchers challenged this statement because they think this may also be affected by framing (Morrison et al., 2012). For example, "smoking may lead to death" and "smoking may lead to lung cancer" refer to two different results. The two sentences' effect on the motivation of behavior change, thereby, may be different.

When considering motivation factors, there is one thing worth mentioning that ethical problems may arise. If application-tracked interventions cannot protect personal data, the intervention may not work as expected. Although this study suggested that application-tracked behavior change has a huge potential for future research, the ethical problem is inevitable. The worries on data revealing may be a barrier to motivate application-tracked behavior change (Stolt et al., 2018).

It is notable that when considering the effect of different motivation factors, "culture" should also be included. The culture here refers to values, beliefs, language, ideas, customs, and behavioral norms shared by a society (Nastasi, 1998). The culture can affect the targeted competencies (Varjas et al., 2005), thus further affecting different motivation factors (Roberts et al., 2009). For example, general weight-loss intervention for general populations often failed for Hispanics (ETHNIC, 2007). Therefore, interventions should be "culturally adjusted" to provide intervention based on a specific culture. However, now most of the motivation factors are studied regarding the results obtained from a small group, which cannot be enlarged to a general condition conceptually.

The literature review suggests that research on factors affecting motivations of behavior change is limited. Also, the motivation factors are often at a general level and not culturally adjusted. To address the two gaps, considering people with different ages and culture may have a different attitude to factors (Gates et al., 2006), this study tends to detect factors which affect application-tracked behavior change interventions for Chinese aged 18 to 25. Then, effective and targeted suggestions for application-tracked behavior intervention designers were promoted based on findings

\section{METHODOLOGY}

A 28-day self-report experiment followed by a focus group was used to detect which factors can affect behavior change motivations. The self-report method allows participants to record whatever is related to digital behavior change (Williams, 2015; Lupton, 2016). The self-report here is in the form of diary. In the diary, participants can record their behavior change experience or anything else they want, like if they achieve the goal or think some factors affect the behavior change.

Table 1. Group division

\begin{tabular}{|l|l|l|}
\hline $\begin{array}{l}\text { Group } \\
\text { number }\end{array}$ & Number of participants & The behavior they want to change \\
\hline Group 1 & 7 (P1,P2,P3,P5,P6,P7,P8) & Drink reminder \\
\hline Group 2 & $6(\mathrm{P} 9, \mathrm{P} 10, \mathrm{P} 11, \mathrm{P} 12, \mathrm{P} 13, \mathrm{P} 41)$ & Drink reminder \\
\hline Group 3 & $9(\mathrm{P} 14, \mathrm{P} 15, \mathrm{P} 16, \mathrm{P} 18, \mathrm{P} 19, \mathrm{P} 20, \mathrm{P} 21, \mathrm{P} 22, \mathrm{P} 23)$ & Sleeping \\
\hline Group 4 & $8(\mathrm{P} 4, \mathrm{P} 24, \mathrm{P} 25, \mathrm{P} 26, \mathrm{P} 27, \mathrm{P} 28, \mathrm{P} 29, \mathrm{P} 49)$ & Sleeping \\
\hline Group 5 & 8 (P30,P31,P32,P33,P34,P36,P37,P38) & Doing exercise \\
\hline Group 6 & 6 (P17,P44,P45,P46,P47,P48) & $\begin{array}{l}\text { Study; Reduce time on bed } \\
\text { Reduce sedentary time; } \text { Lose weight }\end{array}$ \\
\hline Group 7 & 5 (P35,P39,P40,P42,P43) & Sleeping quality; Make a budget \\
\hline
\end{tabular}

The focus group allows researchers to gather more information on the identified factors. The focus groups were generated based on the behaviors that participants want to change. The division is shown in Table 1. Since there are too many participants on drinking and sleeping, to control the discussion, they are divided into two groups respectively, and each group involves fewer than 10 participants (Gibbs, 1997; Chiasson et al., 2015; Sritakaew et al., 2017). Since fewer people in "study, reduce time on bed, reduce sedentary time, lose weight, sleeping quality, make a budget", they are grouped based on the number of participants.

Questions are about the intervention of applications like "Which kind of effect you think your application make on you?", "Can you give some examples?", "Can you describe some good/negative 
points of the application you selected and give some examples?", "Will you keep using this application to change your behavior later?", etc.

\subsection{Participant}

49 Chinese participants ( 23 females, 26 males), aging from 18 to 25 , were recruited in this study. All of them are graduate students in Chinese universities. Participants were those who tend to change their poor health-related behavior and want to use applications as an intervention to change their behavior. The behaviors and the applications are decided by participants as long as the behavior is related with daily health to control the initial self-determination. The behaviors and the applications they selected included changing sleeping time (17) with application RiChangDaKa, HaoShuiMian, JianKangNaoZhong, Sleeping Town, Sleep Cycle, Alarm, Pillow Automatic; drink more water (13) with application HeShuiTiXing, JiShuiZhang, Drink water reminder, Water Reminder - Daily tracker; doing more exercise with application Walkup, YuePaoQuan, GuDong, Keep, Runtastic, Polar, Nike; improving sleeping quality with application WoNiuShuiMian, BaiZaoYin, Snail Sleep; changing studying habits with application Fit Plan, Habit up; losing weight with application Boheyingyangshi,Jishiqi; reducing sedentary time with application Alarm.

The methods they used to record self-report including Memo in smartphones (23), "Word" in computer, iPad or smartphones (14), Diary application (9) and Recorder (3). Table 2 shows when the participants keep the diary. Participants can select if they want to use an alarm. However, this is not compulsory behavior. Finally, 9 people decided to use the alarm or alarm functions to remind them.

\subsection{Protocol}

All participants were voluntarily participating in this experiment. After reading the information sheet and signing the consent form, participants selected the behaviors they wanted to change, the application they planned to use, the method they used to keep a diary, why they want to change this behavior and if they decided to set an alarm/alarms to remind themselves.

In the following 28 days, they need to record data and keep a diary on the behavior change experience. The diary is all in Chinese. The native language can help them express comfortably and accurately.

After 28 days, the 49 participants were divided into 7 groups by their targeted behavior. Participants attended a 1.5-hour focused group discussion in Chinese. Participants were allowed to bring a paper copy of their diary or data to recall what had happened in the 28 self-report studies.

Researchers controlled the time and ensured that participants share their views freely and understandably. All discussions were recorded in the recorder.

\section{RESULTS}

Researchers transcribed all materials into digital. After the translation, all recording was destroyed. Then, Thematic Analysis was used to deal with the Chinese translations and the Chinese diary. Similar factors were grouped by two Chinese researchers. Chinese version documents can ensure researchers understand the meanings more accurately. Nvivo was used to code in this process.

Eight themes mentioned by more than 5 participants were selected, including reminder and alarm (27), award (22), cooperation (17), information (16), competition (14), trust and willingness (10), unplanned events (10), and negative results (9). The inter-rater reliability is $82.15 \%$, which is calculated based on the comparison of absence or presence of a set of themes and frequency of the single theme observation (Roberts et al., 2019)

\subsection{Reminder on screen or alarm}

The reminder effect is controversial. More than half of the participants considered reminder as a method to motivate behavior change. The reminder can also be considered a trigger to receive others' positive feedback and self-satisfactory. For example, when a participant's girlfriend sees the exercise data, he received appreciation from her, which made the participant feel proud (P34).

Although some participants think the reminders or alarms can motivate behavior change, only 2 participants used the reminders or alarms during 28 days. One reason for this is too much information was received on phones, being distracting. Reminders and alarms will bring a burden on dealing information (P26). Therefore, the reminders and alarms, including less information, are more likely to be given up (P35). The balance of real life should be considered. Another reason is the embarrassment 
caused by alarms. For example, P10 set a drinking water reminder, and it alarmed once in class. This embarrassing experience stopped P10 from using the reminders. This is not an isolated case. A similar condition happened to $\mathrm{P} 41$ when she was in public.

7 people think reminders and alarms were useless and annoying. For example, when P32 heard the alarm reminding her to run, she feels it was unnecessary.

The third group of participants has a neural view of the reminders and alarms, yet they tend not to use the alarms or reminders. However, since it can be closed in one second, it will also not harm to motivation (P7). P2 mentioned, "I do not know why every application will ask me if I want to be noticed. Maybe because of the system? Or advantages? I am not sure. But I just need to refuse it. One click of 'not shown on the screen' is not a hard behavior."

\subsection{Award}

There are two types of awards to motivate behavior change: awards from the application and awards from real life.

Award from applications includes figure cultivation, punch card collection, or house building. Participants can obtain motivation from the sense of achievement (P2 in diary). One special award from the application is the refund. Participants will first pay a certain amount of money, and if they can achieve the goal, they would receive a refund. This kind of award can have a stronger effect on motivation because of the sense of loss and willingness to take it back (Group 5 and Group 6).

Real-life awards refer to the improvement like the positive award from changing behavior or participant-defined positive award. Participant-defined positive changes are the award set by participants, which breaches the behavior change like a cheating day when losing weight, one day leaves when studying or a crazy night when trying to change bedtime.

Positive award from changing behavior is the result of changing the behavior. For example, the results of making a budget can lead to a good purchasing habit, while the results of going to bed early can be good for health. This final benefit is different from other awards because its effect on motivation is not a shortly foreseeable award. If the award or benefits are not easy to predict and achieve, it could not provide motivation (P30). For example, "not staying up late would help stay healthy" is the reason why a participant wants to change the behavior. However, it could not motivate the behavior change when a participant wants to give up (P47) because going to bed early will not immediately and physically allow them to feel healthier.

\subsection{Cooperation}

Cooperation can motivate behavior change. Participants in Group 4 mentioned that "If my behavior will affect others' benefit, I will control myself'.P14explainedin the diary "Because I fail to go to bed before $12 \mathrm{am}$, we did not collect the card. It is my fault. I know I harm others' benefit. It is embarrassing and shameful." Also, P37 added in the diary "I feel regretful for my friends since I did not keep my promise to go to the gym with her." Notably, guilty emotions were normally generated because of the harm of others' benefits. P25gave an example in her diary, "I will feel uncomfortable, if the building is not started because of me. (P25 use the application SleepTown ${ }^{1}$.)." P25's opinionis seconded by P47, "Because of drinking Bubble tea in weekdays, my friends and I cannot eat outside on weekends. I was so sorry for her that I did not drink any Bubble tea this week, so we eat outside on that weekend."However, some targets such as doing exercise, making a budget, keeping sedentary, going to bed on time, etc. do not require cooperation. For example, P31 pointed out that "I like to listen to music when running. This is impolite when I run with others. However, if I do not listen to music, I cannot be distracted, and thus the running is hard for me."Therefore, whether the cooperation is a positive or negative effect on motivation depends on habits and behaviors they want to change.

\subsection{Information}

For the information, both positive and negative information can trigger the motivation of behavior change. The reason why information can trigger motivation is that it will bring participants' concern on

1SleepTown is an application which asks one or more people to set the same bedtime. If all people could sleep on time, the application will a build house at night. If there are some people not sleep on time, the house building will be affected. 
self-related benefits. For positive information, after reading an introduction about managing money, P35 gained more motivation to keep making the budget. Simultaneously, the thought about fearing to be poor upon retirement is an example of negative information motivating behavior change.

Comments are a kind of information which can also motive behavior change. However, the effect of comments may be decided by participants. Participants who are more concerned about others' views will be affected by comments more. Take P47 and P17 as two examples. P47 wanted to lose weight because a passenger thought P47 was pregnant and offered a seat to her in the subway, while P17 also received some comments about she should lose weight. However, she does not think she is fat and refuses to do anything to lose weight. People may also predict the commons from others. These predicted comments may also be a kind of motivation. For example, P47 has more motivations to keep studying when thinking being a knowledgeable person will receive praise.

\subsection{Competition}

As a motivation, competition works on doing exercise, losing weight, studying, and making a budget. It always happened with cooperation and spontaneously stimulates the motivation of behavior change. For example, P47 written in diary that, "When I find I take more Calories than my friends, I try to rearrange my menu and generate a lower Calorie menu." However, one limitation of the competition is that the competition may trigger over-motivation, especially for competitive psychology. P32 wrote that "I wanted to rank top, but I was not the first today. Then I walked into my apartment to take me back to the top." Finally, this participant said, "I have to stop because I can do nothing apart from walking." Some people in Group 5 try to explain this condition that over-motivation comes from the eagerness to win and will stop when it harms life.

\subsection{Trust and willingness}

Trust and willingness can affect behavior change if the participants choose to keep using the application. Many factors can affect trust and willingness, like suggested by friends (P14), graphic shown in the application (P33), sense of profession (P46). The reason why these can be the factors is that participants tend to believe experts. Therefore, the evidence from those people may increase the belief that the applications are reliable. Since the application is selected to change the behaviors, "the behavior changing being more easily to achieve" may be taken for granted by participants on some levels.

\subsection{Unplanned event}

"Unplanned event" refers to the events that out of plans. This is a common reason to barrier behavior change. For example, a noisy environment may trigger lazy psychology and give up studying (P47). An invitation to drink may stop people from going to bed early (P23). A shopping may break the nosugar intaking plan (P17). The results of unplanned events can be divided into two. Nearly all participants will generate guilty feelings; some people will transfer this guilty into motivation while others will only consider it as a break of behavior change and do nothing.

\subsection{Negative results}

It is proved that some negative results could trigger motivation. For instance, it shows that a foreseeable disease, will be a motivation to change health-related behavior. To be more specific, nine people (P2, P9, P14, P21, P27, P31, P35, P37, P48) mentioned the behavior change on sleep, exercise, drinking, sleeping quality, weight, bedtime, sedentary can be motivated by related them with the disease. For example, after reading the news sedentary will bring potential risks on legs, P48 walked outside that day spontaneously. When reading an article about studying up late will trigger sudden death, P27 decided to sleep earlier immediately that night. However, how long the change can last is not sure in this study.

\section{DISCUSSION}

From the 28-day self-report and the focus discussion, the researchers summarized 8 Chinese-cultureadjusted factors that may affect application behavior change motivation. The discussion section will compare these factors with existing literature to see if the founded Chinese-culture-adjusted factors are general factors or culture-specific factors. Then, the paper promotes some suggestions in future design 
on application behavior change for Chinese-culture users. Finally, the limitation and future research will be summarized.

\subsection{Comparison with existing research and the implication for design practitioners}

In this session, we compare our findings with with existing research. However, not all founded factors have been fully understood by previous research. Therefore, this section only compared the social connection (cooperation and competition), award, and trust. The three factors are popular mentioned in previous research and also be summarized from this study.

At the same time, the session will discuss how the found factors affect the implication for design practitioners. This will include more factors like self-report, positive effect of negative results and reducing cheating. These factors were not obviously enough as an independent behavior-change motivations, but still worth to be considered as a potential behavior change implication.

Notably, the implications mentioned are based on Chinese aged 18 to 25. Therefore, the implications adopted in this paper may best applicable for this group of users. The effect of these suggested implications on other culture and aged people are not highly supported.

\subsubsection{Award}

Similar with existing papers, our study echoes the previous research that the award from application and participant-defined positive award can motivate behavior change(Conroy et al., 2014; Morrison et al., 2012; Yardley et al., 2016). In addition, our study indicated a possibility why getting refund can be a strong motivation from the sense of losing. However, our study cannot support the positive award from changing the behavior can motivate behavior change(Conroy et al., 2014; Yang et al., 2015).

\subsubsection{Cooperation}

For the cooperation, thirteen participants' results paper supports cooperation can motivate behavior change(Conroy et al., 2014; Lathia et al., 2013; Mohr et al., 2011; Morrison et al., 2012; Schueller et al., 2013). However, the study also promoted a possibility that the a fear of harming others may make the cooperation less motivated (Yardley et al., 2016). However, because of the effect from other interventions, if it is a decisive factor remains unsure (Marshall et al., 2003; Park et al., 2008). Also, this paper suggested that the effect of cooperation should be based on both the changing behaviors and personality of participants instead of simply group participants with the behaviors they want to change. Under this condition, the paper suggested that the cooperation should be carefully designed. An option that can allow users to decide if they want to cooperate spontaneously may be a better choice.

\subsubsection{Competition}

The paper supports the statement that competition could motivate behavior change (Lathia et al., 2013; Morrison et al., 2012). The paper also pointed out that the positive effect on competition may only generate in a limited range of behavior changes, like doing exercise, losing weight, studying, and making budgets. Also, this study promoted a possibility of over-motivation triggered by competition. This is also consistent with the existing study that strict participants may be harmed in health and benefits by the competition (Piccione, 1978). Therefore, when considering using competition as a motivation, the intervention designers should consider an upper limit mechanism. This mechanism may smartly identify abnormal data.

\subsubsection{Trust}

We get the same result agreeing with previous research that professional suggestions can increase the application's reliability (Patten et al., 2007; Yardley et al., 2016). In addition, the paper added two other ways to increase trust - recommend by friends and professional data expression.

An interesting result is that from the results, there are very few people ( 2 participants) spontaneously mentioned the worrying on personal data protection. This is different from the exiting general consideration condition in America or Europe (Bennett, 1992). This difference has happened maybe because, on the one hand, the participants' group may have a high trust for applications or do not think the data that applications have recorded can invade their life. On the other hand, there may be a cultural difference among Chinese and America or Europe (ETHNIC, 2007). 


\subsubsection{Diary area}

Among participants, 35 of them supported that self-report is a method to motivate behavior change (Conroy et al., 2014), because a diary can help them recall what has happened yesterday and introspect themselves. Thereby, only data recording may be not enough. Adding a recalling diary function as an easy method is a potential attempt. However, 9 participants refused to use a diary because they think dairy keeping is a childish, annoying, or bothering behavior. Therefore, an easy-recording and funny diary needs considering by intervention designers is needed, such as linking keeping diary behaviors with unlocking puzzles or building a digital house.

\subsubsection{Negative results}

Although commonly thought of negative results is that it would have a weak motivation effect (Yardley et al., 2016), some existing research has mentioned that unhappy emotions and negative results on health may be a good motivation for behavior changes (Norem and Cantor, 1986). However, this factor is less used in behavior change applications. In other words, some potential negative effects may be shown as a reminder to motivate participants (Conroy et al., 2014; Lathia et al., 2013). 9 participants agree on this. For example, "keeping sedentary for a long time will make your waist uncomfortable" (P41). If the application could be smarter, for example, the application can identify the negative words and provide some notice in a diary like "you fail to go to bed early in three days", the participants can be motivated by this announcement. In this way, the behavior may be more easily to be changed. Or if the applications can remind the negative feelings like "you have be in sedentary for 4 hours. If you do not have a rest and leave your seat, you may feel uncomfortable at the end of the day."

\subsubsection{Data Cheating}

In this study, 41 participants have the experience to try to cheat or ignore negative effects.. An example of ignoring negative effects is that P47 ignored that he has eaten a snack before dinner. As for cheating, P22, who used sleep Town to track bedtime change, admitted that when work is not finished by the target bedtime, he would simply click sleeping in his phone ${ }^{2}$ and keep working on his laptop. Similar conditions happened to P34, who waved his phone to increase the walking steps and cheated the application that he has walked enough steps.

This cheating behavior was also mentioned by other researchers (Hermsen et al., 2016). However, it is notable that this may be a culturally embedded issue. The citations used appear to be from Englishspeaking cultures, yet all participants are from China in this study, so "culturally adjusted" may be one reason why cheating is less mentioned from other culture's participants.

Based on above, the exiting of substitutes and single data collecting measurement is not enough to get the real data. A smarter method to distinguish cheating data is needed. Maybe more sensors can be used. For example, it would work better to identify whether users sleep by detecting the illumination intensity. Also, if the designers tend to design an international application-tracked intervention, the interventions may need to be adjustable across cultures.

\subsection{Limitation and future research}

The self-report methods used in this study can be limited. Although this method can be used to report participants' thoughts, feedback, and experience, as this paper shows, self-report can trigger participants' introspection and motivate behavior change. Therefore, it is important to detect in which levels the self-report can affect behavior change in Chinese culture.

In addition, cheating in a diary may reduce the reliability of the study. To mitigate this limitation, we ask participants if they have included some cheating data in their diary and remembered which data is cheating data. However, if the participants admitted the cheating data or remembered, all cheating data cannot be guaranteed. The cheating data may also happen in the focus group. Some shy participants may not share their different opinions. They may agree on some statements in the real-world group interview while they do not agree on it in their mind. In addition, the subconscious memory error may also cause unintended cheating. Self-report can only ask participants to record their daily life. The accuracy of the data will be higher than a simple after-study report. However, since it is not instantly recording, some

2When the sleeping button switch on, users could not use the phone unless they close the sleeping condition 
memory related to the behavior change may still be vague. The ideal information instead of real condition will replace the vague memory when keeping a diary and lead to unintended cheating.

Furthermore, this study focused on the factors that may motivate the behavior change from self-report. The paper ignores the investigation of the behavioral change is objective or not. In this study, if the behavior has changed successfully is only perceived by participants subjectively, so some participants may overstate or narrow the real condition. This will reduce the reliability of the motivating factors. In the future, an objective method to reflect whether the behavior changes successfully is needed.

For future research, although this study suggested that application-tracked behavior change has a huge potential. The ethical problem is inevitable. The worries on data revealing may be a barrier to motivate application-tracked behavior change. This is not shown in (Stolt et al., 2018).

\section{CONCLUSION}

The research analyses 18 to 25 -year-old Chinese people's motivations on application-tracked behavior change by a daily feedback self-reporting recording and focused group. The paper identifies 8 factors that can affect motivations of behavior change through applications. Based on these factors, the research mainly identified how competition and negative results can affect the 18 to 25-year-old Chinese people's motivation of behavior change. In addition, the paper further explained why some statements on the same factors are controversial. At the same time, this paper promoted six interventions to increase the positive effect of behavior change applications.

\section{REFERENCES}

Conroy, D. E., Yang, C.-H. and Maher, J.P. (2014), "Behavior change techniques in top-ranked mobile apps for physical activity", American Journal of Preventive Medicine, Vol. 46 No. 6, pp.649-652. https://doi.org/10.1016/j.amepre.2014.01.010

Chiasson, K., Terras, K., and Smart, K. (2015), "Faculty perceptions of moving a face-to-face course to online instruction", Journal of College Teaching and Learning (TLC), Vol.12 No.3, pp.321-240. https://doi.org/10.19030/tlc.v12i3.9315

Elaheebocus, S. M. R. A., Weal, M., Morrison, L. and Yardley, L. (2018), "Peer-based social media features in behavior change interventions: systematic review", Journal of Medical Internet Research, Vol.20 No.2, pp. e20. https://doi.org/10.2196/jmir.8342

Gibbs, A. (1997). "Focus groups". Social research update, Vol.19 No.8, pp.1-8.

Hermsen, S., Frost, J., Renes, R. J. and Kerkhof, P. (2016), "Using feedback through digital technology to disrupt and change habitual behavior: A critical review of current literature", Computers in Human Behavior, Vol. 57, pp. 61-74. https://doi.org/10.1016/j.chb.2015.12.023

Lathia, N., Pejovic, V., Rachuri, K. K., Mascolo, C., Musolesi, M. and Rentfrow, P. J. (2013), "Smartphones for large-scale behavior change interventions", IEEE Pervasive Computing, Vol.12 No. 3, pp.66-73. https://doi.org/10.1109/mprv.2013.56

Lorig, K. R., Ritter, P. L., Laurent, D. D., Plant, K. J. A. C. and Rheumatology, R. (2008), "The internet-based arthritis self-management program: A one-year randomized trial for patients with arthritis or fibromyalgia", Arthritis \& Rheumatism, Vol.59 No.7, pp. 1009-1017. https://doi.org/10.1002/art.23817

Lupton, D. (2016), The quantified self, John Wiley and Sons.

Marshall, A. L., Leslie, E. R., Bauman, A. E., Marcus, B. H. and Owen, N. (2003),"Print versus website physical activity programs: a randomized trial", American Journal of Preventive Medicine, Vol.25 No. 2, pp.88-94. https://doi.org/10.1016/s0749-3797(03)00111-9

McGloin, A. F. and Eslami, S. (2015),"Digital and social media opportunities for dietary behaviour change", Proceedings of the Nutrition Society, Vol.74 No. 2, pp. 139-148. https://doi.org/10.1017/s0029665114001505

Michie, S., Yardley, L., West, R., Patrick, K. and Greaves, F. (2017), "Developing and evaluating digital interventions to promote behavior change in health and health care: recommendations resulting from an international workshop", Journal of Medical Internet Research, Vol.19 No.6, pp. e232. https://doi.org/10.2196/jmir.7126

Mohr, D., Cuijpers, P. and Lehman, K. (2011),"Supportive accountability: a model for providing human support to enhance adherence to eHealth interventions", Journal of Medical Internet Research, Vol. 13 No.1, pp. e30. https://doi.org/10.2196/jmir.1602

Morrison, L. G. (2015), "Theory-based strategies for enhancing the impact and usage of digital health behaviour change interventions: a review", DIGITAL HEALTH, Vol.1, pp. 2055207615595335. https://doi.org/10.1177/2055207615595335 
Morrison, L. G., Yardley, L., Powell, J., Michie, S. (2012), "What design features are used in effective e-health interventions? A review using techniques from critical interpretive synthesis", Telemedicine and e-Health, Vol.18 No.2, pp. 137-144. https://doi.org/10.1089/tmj.2011.0062

Norem, J. K., Cantor, N. (1986), "Defensive pessimism: Harnessing anxiety as motivation", Journal of Personality and Social Psychology, Vol.51 No.6, pp.1208. https://doi.org/10.1037/0022-3514.51.6.1208

Park, A., Nitzke, S., Kritsch, K., Kattelmann, K., White, A., Boeckner, L., Lohse, B., Hoerr, S., Greene, G., Zhang, Z. (2008), "Internet-based interventions have potential to affect short-term mediators and indicators of dietary behavior of young adults", Journal of Nutrition Education and Behavior, Vol.40 No.5, pp. 288297. https://doi.org/10.1016/j.jneb.2008.02.001

Patrick, K., Hekler, E. B., Estrin, D., Mohr, D. C., Riper, H., Crane, D., Godino, J. and Riley, W. T. (2016), "The pace of technologic change: implications for digital health behavior intervention research", American Journal of Preventive Medicine,Vol.51 No. 5, pp. 816-824. https://doi.org/10.1016/j.amepre.2016.05.001

Patten, C. A., Rock, E., Meis, T. M., Decker, P. A., Colligan, R. C., Pingree, S., Dornelas, E. A., Offord, K. P., Boberg, E. W. and Gustafson, D. H. (2007), "Frequency and type of use of a home-based, Internet intervention for adolescent smoking cessation", Journal of Adolescent Health, Vol.41 No. 5, pp. 437-443. https://doi.org/10.1016/j.jadohealth.2007.05.016

Piccione, A. (1978), The effects of success-contingent versus engagement-contingent rewards on multiple indices of intrinsic motivation, Doctoral dissertation, Bowling Green State University.

Rooksby, J., Rost, M., Morrison, A., and Chalmers, M. (2014), "Personal tracking as lived informatics", In Proceedings of the SIGCHI conference on human factors in computing systems, pp. 1163-1172. https://doi.org/10.1145/2556288.2557039

Roberts, K., Dowell, A., and Nie, J. B. (2019), "Attempting rigour and replicability in thematic analysis of qualitative research data; a case study of codebook development", BMC medical research methodology, Vol.19 No.66, pp.1-8. https://doi.org/10.1186/s12874-019-0707-y

Ryan, R. M.,and Deci, E. L. (2000), "Self-determination theory and the facilitation of intrinsic motivation, social development, and well-being", American psychologist, Vol.55 No.1, pp.68-78. https://dx.doi.org/10.1037110003-066X.55.1.68

Schueller, Stephen M., Ricardo F. Muñoz, and David C. Mohr. (2013), "Realizing the potential of behavioral intervention technologies." Current Directions in Psychological Science, Vol.22 No.6, pp. 478-483. https://doi.org/10.1177/0963721413495872

Spittaels, H., De Bourdeaudhuij, I., Brug, J., and Vandelanotte, C. (2007), "Effectiveness of an online computertailored physical activity intervention in a real-life setting", Health education research, Vol.22 No.3, pp. 385-396. https://doi.org/10.1093/her/cyl096

Sritakaew, N., O'Brien, A. P., and Hoffman, K. (2017), "Nursing the family of teenage mothers in Thailand: Under pressure and the lack of support", Australian Nursing and Midwifery Journal, Vol.24 No.11,pp. 39.

Williams, K. (2015),"An anxious alliance", In Proceedings of The Fifth Decennial Aarhus Conference on Critical Alternatives, pp. 121-131. https://doi.org/10.7146/aahcc.v1i1.21146

Yang, C. H., Maher, J. P., \& Conroy, D. E. (2015), "Implementation of behavior change techniques in mobile applications for physical activity", American journal of preventive medicine, Vol.48 No.4, pp. 452-455. https://doi.org/10.1016/j.amepre.2014.10.010

Yardley, L., Douglas, E., Anthierens, S., Tonkin-Crine, S., O’Reilly, G., Stuart, B., Geraghty, A. W., ArdenClose, E., van der Velden, A. W. and Goosens, H. (2013), Evaluation of a web-based intervention to reduce antibiotic prescribing for LRTI in six European countries: quantitative process analysis of the GRACE/INTRO randomised controlled trial, Implementation Science, Vol. 8 No.134. https://doi.org/10.1186/1748-5908-8-134

Yardley, L., Morrison, L., Bradbury, K. and Muller, I. (2015), "The person-based approach to intervention development: application to digital health-related behavior change interventions", Journal of medical Internet research, Vol.17 No.1, pp. e30. https://doi.org/10.2196/jmir.4055

Yardley, L., Spring, B. J., Riper, H., Morrison, L. G., Crane, D. H., Curtis, K., Merchant, G. C., Naughton, F. and Blandford, A. (2016), "Understanding and promoting effective engagement with digital behavior change interventions", American journal of preventive medicine, Vol.51 No. 5, pp. 833-842. https://doi.org/10.1016/j.amepre.2016.06.015 